\title{
A CLASS OF IRREDUCIBLE MATRIX REPRESENTATIONS OF AN ARBITRARY INVERSE SEMIGROUP
}

\author{
by W. D. MUNN
}

(Received 4 October, 1960)

By a ' representation' we shall mean throughout a representation by $n \times n$ matrices with entries from an arbitrary field. Elsewhere [9] the author has introduced the concept of a principal representation of a semigroup $S$ (see $\S 3$ below for the definition) and has shown that if $S$ satisfies the minimal condition on principal ideals then every irreducible representation is of this type. Moreover, if $S$ satisfies the minimal conditions on both principal left and right ideals, which together imply the minimal condition on principal two-sided ideals $[6$, Theorem 4], the irreducible representations of $S$ can ultimately be expressed explicitly in terms of group representations.

For an arbitrary semigroup, however, there may exist irreducible representations which are not principal and a different approach is required. The purpose of this paper is to begin a study of representations of semigroups without minimal conditions. We confine our attention here to inverse semigroups.

As a first step, we examine certain congruences on an inverse semigroup $S$. It is shown in Theorem 1 that there is a congruence $\sigma$ on $S$ such that (i) $S / \sigma$ is a group and (ii) if $\tau$ is any congruence on $S$ such that $S / \tau$ is a group then every $\tau$-class is a union of $\sigma$-classes; that is, $\sigma \subseteq \tau$. Thus every group which is a homomorphic image of $S$ is isomorphic with a quotient group of $S / \sigma$ : we denote $S / \sigma$ by $G_{S}$ and call it the maximal group homomorphic image of $S$.

A representation $\Gamma$ of a semigroup is termed prime if the set $V(\Gamma)$ on which it vanishes is either a prime ideal or the empty set. In Theorem 2 we determine all the prime irreducible representations of an inverse semigroup $S$. If $\Gamma$ is such a representation, the complement of $V(\Gamma)$ in $S$ is itself an inverse semigroup and $\Gamma$ is essentially an irreducible representation of its maximal group homomorphic image. While every inverse semigroup has at least one prime irreducible representation, an irreducible representation need not be prime.

The last section of the paper is concerned with a semigroup $S$ with the property that $a \in S a^{2} S$ for all $a \in S$; such a semigroup is called intraregular and, in the terminology of [3], may be characterised as a semilattice of simple semigroups. We show that every irreducible representation of an intraregular semigroup $S$ is prime (Theorem 3); here $S$ does not require to be an inverse semigroup. From Theorems 2 and 3 we may obtain all the irreducible representations of an intraregular inverse semigroup. In particular, the results apply to the interesting case of a semilattice of groups: we conclude by deducing the form of the principal irreducible representations of such a semigroup and an example is given of non-principal irreducible representations.

1. The maximal group homomorphic image of an inverse semigroup. A semigroup is a set which is closed under an associative binary operation: this will, as usual, be denoted by juxtaposition.

Let $S$ be a semigroup and let $a \in S$. By an inverse of $a$ we shall mean an element $a^{\prime} \in S$ such that

$$
a a^{\prime} a=a, \quad a^{\prime} a a^{\prime}=a^{\prime} .
$$


This is a natural extension of the concept of a group inverse, but in general when such an element $a^{\prime}$ exists, it will not be uniquely determined by $a$. The elements $a a^{\prime}$ and $a^{\prime} a$ are evidently idempotents, but they need not be equal. We say that $S$ is regular if and only if $a \in a S a$ for every $a \in S$ : it is not difficult to see that if this is the case then every element of $S$ possesses an inverse in the above sense.

The following three properties of a semigroup $S$ are equivalent $[7,10]$.

P1. $S$ is regular and its idempotents commute.

P2. Every element of $S$ has a unique inverse.

P3. Each principal left ideal and each principal right ideal of $S$ is generated by one and only one idempotent.

A semigroup with these properties is called an inverse semigroup. Such semigroups were first studied by Vagner [15] (under the title 'generalised groups ') and, independently, by Preston [11], in both cases with property $\mathrm{Pl}$ as the definition; this property is the one which we shall use most frequently. The unique inverse of $a$ in $S$ is denoted by $a^{-1}$. Then it may readily be shown that $\left(a^{-1}\right)^{-1}=a$ and that $(a b)^{-1}=b^{-1} a^{-1}$ for any elements $a, b \in S$; furthermore, if $e$ is an idempotent of $S$, then $e^{-1}=e$. Inverse semigroups retain certain of the features of groups and have been examined in some detail; an account is given in [4].

Notation. The empty set will be denoted by $\square$. If $B$ is a subset of a set $A$ then $A \backslash B$ will denote the set-complement of $B$ in $A$.

THEOREM 1. Let $S$ be an inverse semigroup and let a relation $\sigma$ be defined on $S$ by the rule that $x \sigma y$ if and only if there is an idempotent $e \in S$ such that ex $=e y$. Then $\sigma$ is a congruence and $S / \sigma$ is a group. Further, if $\tau$ is any congruence on $S$ with the property that $S / \tau$ is a group then $\sigma \subseteq \tau$ and so $S / \tau$ is isomorphic with some quotient group of $S / \sigma$. We denote the maximal group homomorphic image $S / \sigma$ by $G_{\mathbf{S}}$.

If $M$ is an ideal of $S$, then $M$ is an inverse semigroup and $G_{M} \cong G_{S}$.

Proof. It is clear that $\sigma$ is reflexive and symmetric. Suppose that $x, y$ and $z$ are elements of $S$ such that $x \sigma y$ and $y \sigma z$. Then there exist idempotents $e$ and $f$ in $S$ such that $e x=e y$ and $f y=f z$. But since $e f=f e$, ef is also an idempotent and $e f x=f e x=f e y=e f y=e f z$; hence $x \sigma z$ and therefore $\sigma$ is transitive.

Now let $x \sigma y(x, y \in S)$ and let $z$ be any element of $S$. To establish that $\sigma$ is a congruence we have only to show that $(x z) \sigma(y z)$ and that $(z x) \sigma(z y)$. By hypothesis, there is an idempotent $e \in S$ such that $e x=e y$ : thus $e x z=e y z$ and so $(x z) \sigma(y z)$. For the second part, consider the idempotent $(z e)(z e)^{-1}=z e z^{-1}$. We have

$$
\left(z e z^{-1}\right) z x=z z^{-1} z e x=z z^{-1} z e y=\left(z e z^{-1}\right) z y,
$$

which shows that $(z x) \sigma(z y)$.

We next prove that the quotient semigroup $S / \sigma$ is a group. For any $x \in S$ let $x_{\sigma}$ denote the $\sigma$-class of $S$ containing $x$; thus $x \rightarrow x_{\sigma}$ is the natural homomorphism of $S$ onto $S / \sigma$. If $e$ and $f$ are idempotents in $S$, it follows from the relation $(e f) e=(e f) f$ that $e_{\sigma}=f_{\sigma}$; that is, all the idempotents belong to the same $\sigma$-class, which we denote by 1 . Then

$$
1 x_{\sigma}=\left(x x^{-1}\right)_{\sigma} x_{\sigma}=\left(x x^{-1} x\right)_{\sigma}=x_{\sigma}=x_{\sigma}\left(x^{-1} x\right)_{\sigma}=x_{\sigma} 1 \text {; }
$$


also

$$
\left(x^{-1}\right)_{\sigma} x_{\sigma}=\left(x^{-1} x\right)_{\sigma}=1=\left(x x^{-1}\right)_{\sigma}=x_{\sigma}\left(x^{-1}\right)_{\sigma}
$$

and therefore $S / \sigma$ is a group.

Let $\tau$ be a congruence on $S$ with the property that $S / \tau$ is a group. We shall show that if two elements of $S$ lie in the same $\sigma$-class they must lie in the same $\tau$-class. Let the $\tau$-class of $S$ containing $x$ be denoted by $x_{\mathrm{r}}$. Suppose that $x \sigma y(x, y \in S)$. Then there is an idempotent $e$ in $S$ such that $e x=e y$ and so $e_{\tau} x_{\tau}=e_{\tau} y_{\tau}$. But $e_{\tau}$ is an idempotent of the group $S / \tau$ and so it is the identity. Hence $x_{\tau}=y_{\tau}$ which shows that $\sigma \subseteq \tau$. It follows that $S / \tau$ is isomorphic with some quotient group of $S / \sigma$.

Finally, let $M$ be an ideal of $S$ and let $a \in M$. Then since $a^{-1}=a^{-1} a a^{-1} \in M$ we see that $M$ is regular; since, a fortiori, its idempotents commute, it is an inverse semigroup. Let the relation $\mu$ be defined on $M$ by analogy with $\sigma$ on $S$ : we write $x \mu y(x, y \in M)$ if and only if there is an idempotent $e \in M$ such that $e x=e y$; then $G_{M}=M / \mu$. To show that $G_{M} \cong G_{S}$ it is sufficient to prove that every $\sigma$-class of $S$ contains one and only one $\mu$-class of $M$. Let $x \in S$ and let $e$ be an idempotent in $M$; then since $e x=e(e x)$ we see that $x \sigma(e x)$. But $e x \in M$ and so $x_{\sigma} \cap M \neq \square$. We complete the proof by showing that $x_{\sigma} \cap M$ is a $\mu$-class of $M$. If $y, z \in x_{\sigma} \cap M$ there is an idempotent $f \in S$ such that $f y=f z$. Let $e$ be any idempotent in $M$; then $e f$ is an idempotent in $M$ and from the relation $e f y=e f z$ we have $y \mu z$. Conversely, if two elements of $M$ belong to the same $\mu$-class then, a fortiori, they belong to the same $\sigma$-class of $S$.

For the remainder of the paper we shall write $\bar{x}$ in place of $x_{\sigma}$; thus the natural homomorphism of an inverse semigroup onto its maximal group homomorphic image will be denoted by $x \rightarrow \bar{x}$.

Suppose that an inverse semigroup $S$ has a kernel $K$ (a minimal ideal, necessarily unique) and that $K$ is a group. Then it is clear from the last part of the theorem that $G_{s} \cong K$. In fact it may easily be verified that $\bar{x}=\bar{y}(x, y \in S)$ if and only if $e x=e y$, where $e$ is the identity of $K$, and that

$$
\bar{x} \rightarrow e x \quad(x \in S)
$$

is an isomorphism of $G_{S}$ onto $K$. This is a special case of a result due to Stoll [14, p. 481].

An alternative, if less direct proof of the first part of Theorem 1 may be outlined as follows. $\dagger$ An inverse semigroup $S$ is isomorphic with a certain subsemigroup $\Sigma$ of the semigroup of all one-to-one partial transformations of itself $[15 ; 12$, Theorem 1]. Since $\Sigma$ does not contain the null transformation, we may define a congruence $\sim$ on it in the manner described by Rees [13] and the quotient semigroup $\Sigma / \sim$ is a group [13, Lemma 1.23]. The maximality of $\Sigma / \sim$ amongst the group homomorphic images of $\Sigma$ was proved by Stoll [14, p. 479].

2. Prime irreducible representations of an inverse semigroup. Let $\Phi$ be a field and $n$ a positive integer. We shall denote by $\Phi_{n}$ the set of all $n \times n$ matrices over $\Phi$. With respect to the usual matrix operations of addition, multiplication and scalar multiplication $\Phi_{n}$ is a simple algebra over $\Phi$. Let $S$ be a semigroup. By a representation of $S$ of degree $n$ over $\Phi$ we shall mean a homomorphism of $S$ into the multiplicative semigroup of $\Phi_{n}$. The term 'irreducible', as applied to a set of matrices or to a representation, will have its customary meaning. By convention, we shall not regard the set consisting of the $1 \times 1$ zero matrix as irreducible; thus we shall not regard the null representation of degree 1 as an irreducible representation. It should

$\dagger$ Another proof (unpublished) has been obtained by Dr G. B. Preston. 
be noted that since $S$ need not have an identity element there is no counterpart of the familiar additional requirement for group representations that the image of the identity should be the identity matrix. It is frequently the case with a representation $\Gamma$ of $S$ that $\Gamma(x)$ is a singular matrix for all $x \in S$, even if $\Gamma$ is irreducible.

In the proofs of Theorems 2 and 3 we shall make use of the following result from classical representation theory. (See, for example, [4, Chapter V, Theorem 5.7]).

LEMMA. An irreducible subalgebra of $\Phi_{n}$ is a simple algebra over $\Phi$.

The following notation will be used below. Let $T$ be a subset of a semigroup $S$ and let $\Gamma$ be a representation of $S$ of degree $n$ over $\Phi$. Then $\Gamma(T)$ will denote the set $\{\Gamma(t): t \in T\}$ and $[\Gamma(T)]$ will denote the subspace of $\Phi_{n}$ spanned by $\Gamma(T)$. It is easy to see that $[\Gamma(S)]$ is a subalgebra of $\Phi_{n}$ and that if $T$ is an ideal of $S$ then $[\Gamma(T)]$ is an ideal of $[\Gamma(S)]$. We observe that $\Gamma(T)$ is an irreducible matrix set if and only if the same is true of $[\Gamma(T)]$. As in [9] we shall write

$$
V(\Gamma)=\{x: x \in S, \Gamma(x)=0\} .
$$

Either $V(\Gamma)=\square$ or it is an ideal of $S$. If $V(\Gamma)=\square$ or is a prime ideal of $S$ (that is, if $V(\Gamma) \neq S$ and $S \backslash V(\Gamma)$ is a subsemigroup of $S$ ) we shall call $\Gamma$ a prime representation of $S$. Theorem 2 characterises all prime irreducible representations of an arbitrary inverse semigroup.

THEOREM 2. Let $S$ be an inverse semigroup and $\Phi$ a field.

(i) Let $\Gamma$ be a prime irreducible representation of $S$ over $\Phi$ and let $V=V(\Gamma)$. Then $S \backslash V$ is an inverse semigroup and

$$
\Gamma(x)=\left\{\begin{array}{cll}
\Gamma^{*}(\bar{x}) & \text { if } & x \in S \backslash V, \\
0 & \text { if } & x \in V,
\end{array}\right.
$$

where $x \rightarrow \bar{x}$ is the natural homomorphism of $S \backslash V$ onto $G_{S \backslash V}$ and $\Gamma^{*}$ is an irreducible representation of $G_{S \backslash V}$.

(ii) Let $V$ be the empty set or a prime ideal of $S$. Then $S \backslash V$ is an inverse semigroup and if $\Gamma^{*}$ is any irreducible representation of $G_{S \backslash V}$ the mapping $\Gamma$, defined by $(2.1)$, is a prime irreducible representation of $S$.

Proof. (i) Let $a \in S \backslash V$ and suppose that $a^{-1} \in V$; then $a=a a^{-1} a \in V$, which is a contradiction. Hence $a^{-1} \in S \backslash V$ and so the semigroup $S \backslash V$ is regular. Since its idempotents commute it is an inverse semigroup.

We define a positive integer $r$ and a subset $M$ of $S \backslash V$ as follows:

$$
r=\min \{\operatorname{rank} \Gamma(a): a \in S \backslash V\}, \quad M=\{a: a \in S \backslash V, \operatorname{rank} \Gamma(a)=r\} .
$$

Let $a \in M, x \in S \backslash V$. Then $a x \in S \backslash V$; also rank $\Gamma(a x) \leqq \operatorname{rank} \Gamma(a)=r$ and so, by the minimality of $r$, rank $\Gamma(a x)=r$. Hence $a x \in M$; similarly $x a \in M$. Thus $M$ is an ideal of $S \backslash V$ and since $S \backslash V$ is an inverse semigroup so also is $M$.

Evidently $1 \leqq r \leqq n$, where $n$ is the degree of $\Gamma$. Suppose that $r<n$; we shall show that this leads to a contradiction. Since $M$ is an inverse semigroup it contains an idempotent $f$, say. Corresponding to $f$, there is a non-singular matrix $P$ over $\Phi$ such that

$$
P^{-1} \Gamma(f) P=\left[\begin{array}{ll}
I_{r} & 0 \\
0 & 0
\end{array}\right]
$$


Let $\Delta$ denote the representation $x \rightarrow P^{-1} \Gamma(x) P$ of $S$. Since $\Gamma$ is irreducible, so also is the equivalent representation $\Delta$. Thus $[\Delta(S)]$ is an irreducible subalgebra of $\Phi_{n}$ and therefore, by the lemma, it is a simple algebra over $\Phi$. But $[\Delta(S)]=[\Delta(S \backslash V)]$ and $[\Delta(M)]$ is a non-zero ideal of $[\Delta(S \backslash V)]$, since $M$ is an ideal of $S \backslash V$. Hence $[\Delta(M)]=[\Delta(S \backslash V)]$, which shows in particular that the matrix set $\Delta(M)$ is irreducible.

Let $g$ be any idempotent in $M$ and let $\Delta(g)=\left[\begin{array}{ll}G_{1} & G_{2} \\ G_{3} & G_{4}\end{array}\right]$, where the partitioning is such that $G_{1}$ is an $r \times r$ block. Then, since

$$
\Delta(f g)=\left[\begin{array}{ll}
G_{1} & G_{2} \\
0 & 0
\end{array}\right], \quad \Delta(g f)=\left[\begin{array}{ll}
G_{1} & 0 \\
G_{3} & 0
\end{array}\right] \text { and } f g=g f,
$$

we see that $G_{2}=0$ and $G_{3}=0$. But $\Delta(f g)$ is an idempotent matrix of rank $r$ and so $G_{1}=I_{r}$. Thus $\Delta(g)=\left[\begin{array}{cc}I_{r} & 0 \\ 0 & G_{4}\end{array}\right]$ and, since this also has rank $r$, we have $G_{4}=0$.

Now let $a$ be an arbitrary element of $M$ and let $\Delta(a)=\left[\begin{array}{ll}A_{1} & A_{2} \\ A_{3} & A_{4}\end{array}\right]$, with the same partitioning as before. Since $a^{-1} a$ is an idempotent in $M$, we have

$$
\Delta(a)=\Delta(a) \Delta\left(a^{-1} a\right)=\left[\begin{array}{ll}
A_{1} & A_{2} \\
A_{3} & A_{4}
\end{array}\right]\left[\begin{array}{ll}
I_{r} & 0 \\
0 & 0
\end{array}\right]=\left[\begin{array}{ll}
A_{1} & 0 \\
A_{3} & 0
\end{array}\right],
$$

which shows that $\Delta(M)$ is a reducible matrix set. But this contradicts our previous conclusion and so we must have $r=n$. In particular, $\Gamma(e)=I_{n}$ for every idempotent $e \in S \backslash V$. Thus if $x$ and $y$ are elements of $S \backslash V$ such that $e x=e y$ for some idempotent $e \in S \backslash V$, then $\Gamma(x)=I_{n} \Gamma(x)$ $=\Gamma(e x)=\Gamma(e y)=I_{n} \Gamma(y)=\Gamma(y)$. This enables us to define a mapping $\Gamma^{*}$ of $G_{S \backslash V}$ into $\Phi_{n}$ by the rule that $\Gamma^{*}(\bar{x})=\Gamma(x)$. Since $\Gamma^{*}(\bar{x}) \Gamma^{*}(\bar{y})=\Gamma(x) \Gamma(y)=\Gamma(x y)=\Gamma^{*}(\overline{x y})=\Gamma^{*}(\bar{x} \cdot \bar{y})$, $\Gamma^{*}$ is a representation of $G_{S \backslash V}$; further, since $\Gamma^{*}\left(G_{S \backslash V}\right)=\Gamma(S \backslash V)$ and $\Gamma$ is irreducible, the same is true of $\Gamma^{*}$.

(ii) As in the proof of part (i), $S \backslash V$ is an inverse semigroup. Let $\Gamma^{*}$ be an irreducible representation of $G_{S W}$ over $\Phi$ and let $\Gamma$ be defined on $S$ by (2.1). Then, for any elements $x, y \in S \backslash V$ we have

$$
\Gamma(x) \Gamma(y)=\Gamma^{*}(\bar{x}) \Gamma^{*}(\bar{y})=\Gamma^{*}(\bar{x} \cdot \bar{y})=\Gamma^{*}(\overline{x y})=\Gamma(x y) .
$$

Since $\Gamma(x) \Gamma(y)=0=\Gamma(x y)$ if either $x$ or $y$ (or both) $\in V$, we see that $\Gamma$ is a representation of $S$ with $V(\Gamma)=V$. Since $\Gamma(S \backslash V)=\Gamma^{*}\left(G_{S, V}\right)$ and $\Gamma^{*}$ is irreducible, so also is $\Gamma$.

Every semigroup $S$ has a trivial prime irreducible representation, namely the representation $\Gamma$ of degree 1 defined by $\Gamma(x)=1$ for all $x \in S$. However, an irreducible representation of an inverse semigroup need not be prime. To illustrate this, consider the subset $S$ of $\Phi_{n}$ consisting of the $n^{2}$ matrices $E_{i j}$, where $E_{i j}$ is the matrix with 1 in the $(i, j)$ th position and 0 elsewhere, together with the zero matrix. Then it is easy to verify that, with respect to matrix multiplication, $S$ is an inverse semigroup and that the self-representation of $S$ is irreducible. But this representation is not prime if $n>1$.

In the next section we discuss an important class of inverse semigroups for which every irreducible representation is prime. 
3. Application to intraregular inverse semigroups. By a semilattice we mean a commutative semigroup of idempotents. We define a partial ordering in a semilattice $Y$ by writing $e \geqq f(e, f \in Y)$ if and only if $e f=f$ : any two elements have a unique greatest lower bound with respect to this ordering, namely their product. A semigroup $S$ is called a semilattice of semigroups of type $\mathscr{T}[3, \mathrm{p} .499]$ if it is expressible as a union of disjoint subsemigroups $S_{\alpha}$ of type $\mathscr{T}$, where $\alpha$ belongs to a semilattice $Y$ and the multiplication in $S$ is such that $S_{\alpha} S_{\beta} \subseteq S_{\alpha \beta}$.

Following Clifford and Preston [4], we shall say that a semigroup $S$ is intraregular if and only if $a \in S a^{2} S$ for every $a \in S$. Such a semigroup may be characterised as a semilattice of simple semigroups $([1,5]$; see also $[3$, p. 502]). It may be shown that an intraregular inverse semigroup is a semilattice of simple inverse semigroups and conversely.

THEOREM 3. Every irreducible representation of an intraregular semigroup is a prime representation.

Proof. Let $S$ be an intraregular semigroup and let $\Gamma$ be an irreducible representation of $S$ over a field $\Phi$. Let $V=V(\Gamma)$ and let $a, b \in S \backslash V$; to establish the theorem we have to show that $a b \in S \backslash V$. Suppose that this is false and that $a b \in V$. Since $a \in S a^{2} S \subseteq S a S$, the principal ideal of $S$ generated by $a$ is $S a S$. Let $x \in S a S$; there are thus elements $y, z \in S$ such that $x=y a z$. Then since bya $\in(b y a)^{2} S$ and $a b \in V$ we have

$$
b x=b y a z \in S(b y a)^{2} S z=S b y(a b) y a S z \subseteq V .
$$

Hence $\Gamma(b) \Gamma(x)=0$ for all $x \in S a S$ and so $\Gamma(b) X=0$ for all matrices $X \in[\Gamma(S a S)]$. Now $[\Gamma(S a S)]$ is an ideal of $[\Gamma(S)]$ and is non-zero since it contains $\Gamma(a) \neq 0$. But, by the lemma $(\S 2)$, since $\Gamma$ is irreducible, $[\Gamma(S)]$ is a simple algebra. Hence $[\Gamma(S a S)]=[\Gamma(S)]$ and so $[\Gamma(S)]$ contains a non-zero left annihilator, namely $\Gamma(b)$. This contradicts the simplicity of $[\Gamma(S)]$ and completes the proof.

Theorems 2 and 3 taken together enable us to find all the irreducible representations of an intraregular inverse semigroup $S$. We note, in addition, that if $\Gamma_{1}$ and $\Gamma_{2}$ are equivalent irreducible representations of $S$, then $V\left(\Gamma_{1}\right)=V\left(\Gamma_{2}\right)=V$, say, and the corresponding irreducible representations $\Gamma_{1}{ }^{*}$ and $\Gamma_{2}{ }^{*}$ of $G_{S \backslash V}$, defined by (2.1), are also equivalent; conversely, if $\Gamma_{1}{ }^{*}$ and $\Gamma_{2}{ }^{*}$ are equivalent irreducible representations of $G_{S \backslash V}$, where $V=\square$ or is a prime ideal of $S$, then $\Gamma_{1}$ and $\Gamma_{2}$, defined by (2.1), are equivalent irreducible representations of $S$.

Elements of an arbitrary semigroup $S$ are said to be $\mathscr{J}$-equivalent ( $f$-equivalent, in the notation of Green [6]) if they generate the same principal two-sided ideal of $S$ : evidently if $M$ is an ideal of $S$ and $J$ a $\mathscr{J}$-class then either $J \subseteq M$ or $J \cap M=\square$. The $\mathscr{J}$-classes have a natural partial ordering: for any two $\mathscr{J}$-classes $J$ and $J^{\prime}$, with corresponding principal ideals $P(J)$ and $P\left(J^{\prime}\right)$, we write $J \geqq J^{\prime}$ if and only if $P(J) \supseteq P\left(J^{\prime}\right)$. In [9], the author has defined a principal representation of $S$ to be a representation $\Gamma$ such that $S \backslash V(\Gamma)$ contains a unique minimal $\mathscr{J}$. class (the apex of $\Gamma$ ) with respect to this partial ordering; a method is then given for obtaining all the principal irreducible representations of $S$ in terms of irreducible representations of the principal factors of $S$ [9, Theorem 1]. It is shown further that if $S$ satisfies the minimal condition on principal ideals then every irreducible representation is principal [9, Theorem 2]. 
We shall now obtain the principal representations of an important type of intraregular inverse semigroup from the results of the present paper.

The three properties of a semigroup $S$ stated below are equivalent.

P4. $S$ is a union of disjoint groups and its idempotents commute.

P5. $S$ is regular and its idempotents are central (that is, if $e$ is an idempotent then ex $=x e$ for all $x \in S$ ).

P6. $S$ is a semilattice of groups.

Clifford $[2,3]$ showed the equivalence of the properties P4 and P6. That P4 and P5 are equivalent was shown by Liber [7]. Since a group is a special case of a simple inverse semigroup, a semilattice of groups is an intraregular inverse semigroup. Alternatively, we may note that P5 implies P1; also, if $a \in S$ then $a=a a^{-1} a$ and so $a=a a^{-1}\left(\left(a a^{-1}\right) a\right)=a a^{-1} a^{2} a^{-1} \in S a^{2} S$.

The structure of a semilattice of groups may be described as follows [2, Theorem 3]. Such a semigroup $S$ is a union of disjoint groups $S_{\alpha}$, where $\alpha$ belongs to a semilattice $Y$. To each pair of elements $\alpha, \beta \in Y$ such that $\alpha>\beta$ there corresponds a homomorphism $\phi_{\alpha \beta}$ of $S_{\alpha}$ into $S_{\beta}$ and these homomorphisms satisfy the transitivity relation

$$
\phi_{\alpha \beta} \phi_{\beta \gamma}=\phi_{\alpha \gamma} \quad(\alpha>\beta>\gamma) .
$$

If we take $\phi_{\alpha \alpha}$ to be the identity automorphism of the group $S_{\alpha}$, then multiplication in $S$ is according to the rule

$$
x_{\alpha} y_{\beta}=\left(x_{\alpha} \phi_{\alpha \gamma}\right)\left(y_{\beta} \phi_{\beta \gamma}\right) \text {. }
$$

where $x_{\alpha}, y_{\beta}$ are elements of $S_{\alpha}, S_{\beta}$, respectively, and $\gamma=\alpha \beta(\alpha, \beta \in Y)$.

It is not difficult to see that the $\mathscr{J}$-classes of $S$ are precisely the groups $S_{\alpha}$ and that their partial ordering qua $\mathscr{J}$-classes coincides with that determined by the semilattice $Y$.

Now suppose that $\Gamma$ is a principal irreducible representation of $S$ over a field $\Phi$. Let $V$ denote $V(\Gamma)$. By Theorem 3, $\Gamma$ is prime; that is, $S \backslash V$ is a subsemigroup of $S$. Hence, since $\Gamma$ is principal, there is an element $\omega \in Y$ such that $S_{\omega}$ is the kernel of $S \backslash V$. But by (1.1) the mapping $\bar{x}_{\alpha} \rightarrow e_{\omega} x_{\alpha}=x_{\alpha} \phi_{\alpha \omega}\left(x_{\alpha} \in S_{\alpha} \subseteq S \backslash V\right)$ is an isomorphism of $G_{S \mid V}$ onto $S_{\omega}$, where $e_{\omega}$ is the identity of $S_{\omega}$. Thus, applying (2.1), we see that there is an irreducible representation $\Gamma^{*}$ of $S_{\omega}$ over $\Phi$ such that

$$
\Gamma\left(x_{\alpha}\right)=\left\{\begin{array}{ccc}
\Gamma^{*}\left(x_{\alpha} \phi_{\alpha \omega}\right) & \text { if } & x_{\alpha} \in S_{\alpha}, \alpha \geqq \omega, \\
0 & \text { if } & x_{\alpha} \in S_{\alpha}, \alpha \omega .
\end{array}\right.
$$

Conversely, if $\omega$ is any element of $Y$ and if $\Gamma^{*}$ is an irreducible representation of $S_{\omega}$ over $\Phi$, then (3.1) defines a principal irreducible representation of $S$ over $\Phi$, with apex $S_{\omega}$.

If $S$ satisfies the minimal condition on principal ideals then every irreducible representation is principal and therefore is of the type described in (3.1). The particular case in which $S$ is finite was treated in an earlier paper by the author [8, Theorem 9.6].

Finally, as an almost trivial example to illustrate the occurrence of non-principal irreducible representations, consider the semigroup $S$ which consists of the real interval $[0,1]$ with multiplication defined by

$$
x y=\min \{x, y\} .
$$


This is a semilattice in which the ordering is the natural ordering of real numbers. Every proper ideal of $S$ is prime and if $V=\square$ or is a proper ideal then $G_{S \mid V}$ is the group consisting of one element. It is easy to verify that the principal irreducible representations of $S$ over $\Phi$ are the representations $\Gamma_{a}$ of degree 1 given by

$$
\Gamma_{a}(x)= \begin{cases}1 & \text { if } x \geqq a, \quad(0 \leqq a \leqq 1) . \\ 0 & \text { otherwise, }\end{cases}
$$

On the other hand the non-principal irreducible representations are the representations $\Delta_{a}$ of degree 1 given by

$$
\Delta_{a}(x)=\left\{\begin{array}{ll}
1 & \text { if } x>a, \\
0 & \text { otherwise, }
\end{array} \quad(0 \leqq a<1)\right.
$$

\section{REFERENCES}

1. O. Andersen, Ein Bericht über die Struktur abstrakter Halbgruppen, Thesis, Hamburg (1952).

2. A. H. Clifford, Semigroups admitting relative inverses, Ann of Math. (2), 42 (1941), 1037-1049.

3. A. H. Clifford, Bands of semigroups, Proc. Amer. Math. Soc., 5 (1954), 499-504.

4. A. H. Clifford and G. B. Preston, The algebraic theory of semigroups, Mathematical Surveys of the American Mathematical Society (Providence, R. I., 1961).

5. R. Croisot, Demi-groupes inversifs et demi-groupes réunions de demi-groupes simples, Ann. Sci. École Norm. Sup. (3), 70 (1953), 361-379.

6. J. A. Green, On the structure of semigroups, Ann. of Math. (2), 54 (1951), 163-172.

7. A. E. Liber, On the theory of generalised groups, Doklady Akad. Nauk SSSR(N.S.), 97 (1954), 25-28 (Russian).

8. W. D. Munn, On semigroup algebras, Proc. Cambridge Phil. Soc., 51 (1955), 1-15.

9. W. D. Munn, Irreducible matrix representations of semigroups. To appear in Quart. J. Math. Oxford Ser. (2).

10. W. D. Munn and R. Penrose, A note on inverse semigroups, Proc. Cambridge Phil. Soc., 51 (1955), 396-399.

11. G. B. Preston, Inverse semi-groups, J. London Math. Soc., 29 (1954), 396-403. 419.

12. G. B. Preston, Representations of inverse semi-groups, J. London Math. Soc., 29 (1954), $411-$ 281-284.

13. D. Rees, On the group of a set of partial transformations, J. London Math. Soc., 22 (1947), 481.

14. R. R. Stoll, Homomorphisms of a semigroup onto a group, Amer. J. Math., 73 (1951), 475-

15. V. V. Vagner, Generalised groups, Doklady Akad. Nauk SSSR (N.S.), 84 (1952), 1119-1122.

THE UNIVERSITY

Glasgow 